hep-th $/ 9709113$

CERN-TH/97-226

September, 1997

\title{
Duality, Central Charges and Entropy of Extremal BPS Black Holes
}

\author{
Laura Andrianopoli ${ }^{1}$, Riccardo D'Auria ${ }^{2}$ and Sergio Ferrara ${ }^{3}$ \\ ${ }^{1}$ Dipartimento di Fisica, Universitá di Genova, via Dodecaneso 33, I-16146 Genova \\ and Istituto Nazionale di Fisica Nucleare (INFN) - Sezione di Torino, Italy \\ 2 Dipartimento di Fisica, Politecnico di Torino, \\ Corso Duca degli Abruzzi 24, I-10129 Torino \\ and Istituto Nazionale di Fisica Nucleare (INFN) - Sezione di Torino, Italy \\ ${ }^{3}$ CERN Theoretical Division, CH 1211 Geneva 23, Switzerland
}

Talk given by S. Ferrara at the "STRINGS 'gr"' Conference, 16-21 June 1997, Amsterdam, The Netherlands

\begin{abstract}
We report on some general results on the physics of extremal BPS black holes in four and five dimensions. The duality-invariant entropy-formula for all $N>2$ extended supergravities is derived. Its relation with the fixedscalar condition for the black-hole "potential energy" wich extremizes the BPS mass is obtained. BPS black holes preserving different fractions of supersymmetry are classified in a U-duality invariant set up. The latter deals with different orbits of the fundamental representations of the exceptional groups $E_{7(7)}$ and $E_{6(6)}$. We comment upon the interpretation of these results in a string and M-theory framework.
\end{abstract}

* Work supported in part by EEC under TMR contract ERBFMRX-CT96-0045 (LNF Frascati, Politecnico di Torino and Univ. Genova) and by DOE grant DE-FGO3-91ER40662 


\title{
Duality, Central Charges and Entropy of Extremal BPS Black Holes
}

\author{
L. Andrianopoli ${ }^{\mathrm{a}}$, R. D'Auria ${ }^{\mathrm{b}}$ and S. Ferrara ${ }^{\mathrm{c}}$ \\ ${ }^{a}$ Dipartimento di Fisica, Università di Genova, via Dodecaneso 33, I-16146 Genova \\ and Istituto Nazionale di Fisica Nucleare (INFN) - Sezione di Torino, Italy \\ ${ }^{\mathrm{b}}$ Dipartimento di Fisica, Politecnico di Torino, \\ Corso Duca degli Abruzzi 24, I-10129 Torino \\ and Istituto Nazionale di Fisica Nucleare (INFN) - Sezione di Torino, Italy
}

${ }^{\mathrm{c}}$ CERN Theoretical Division, CH-1211 Geneva 23, Switzerland

\begin{abstract}
We report on some general results on the physics of extremal BPS black holes in four and five dimensions. The duality-invariant entropy-formula for all $N>2$ extended supergravities is derived. Its relation with the fixedscalar condition for the black-hole "potential energy" wich extremizes the BPS mass is obtained. BPS black holes preserving different fractions of supersymmetry are classified in a U-duality invariant set up. The latter deals with different orbits of the fundamental representations of the exceptional groups $E_{7(7)}$ and $E_{6(6)}$. We comment upon the interpretation of these results in a string and M-theory framework.
\end{abstract}

\section{Introduction}

In recent time, remarkable results have been obtained in the study of general properties of BPS states both in supersymmetric gauge theories as well as in supersymmetric theories of gravity. The latter are described by string theory and M-theory [1] whose symmetry properties are encoded in extended supergravity effective field theories.

Of particular interest are extremal black holes in four and five dimensions which correspond to BPS saturated states [2] and whose ADM mass depends, beyond the quantized values of electric and magnetic charges, on the asymptotic value of scalars at infinity. The latter describe the moduli space of the theory Another physical relevant quantity, which depends only on quantized electric and magnetic charges, is the black hole entropy, which can be defined macroscopically, through the Bekenstein-Hawking area-entropy relation or microscopically, through D-branes techniques [3] by counting of microstates [4]. It has been further realized that the scalar fields, independently of their values at infinity, flow towards the black hole horizon to a fixed value of pure topological nature given by a certain ratio of electric and magnetic charges [5]. These "fixed scalars" correspond to the extrema of the ADM mass in moduli space while the black-hole entropy is the value of the squared ADM mass at this point in $D=4$ [6] [7] and the power $3 / 2$ of the ADM mass in $D=5$. In four dimensional theories with $N>2$, extremal black-holes preserving one supersymmetry have the further property that all central charge eigenvalues other than the one equal to the BPS mass flow to zero for "fixed scalars". This is not true in $D=5$ because the charges transform in the antisymmetric representation of $U \operatorname{sp}(N)$ instead of $U(N)$ as in the four dimensional cases.

The entropy formula turns out to be in all cases a U-duality invariant expression (homogeneous of degree two in $D=4$ and of degree $3 / 2$ in $D=5$ ) built out of electric and magnetic charges and as such can be in fact also computed through certain (moduli-independent) topological quantities which only depend on the nature of the Uduality groups and the appropriate representations of electric and magnetic charges. For example, in the $N=8, D=4$ theory the entropy was shown to correspond to the unique quartic $E_{7}$ invariant built with its 56 dimensional representation [8]. In this report we intend to summarize 
further progress in this subject by deriving, for all $N>2$ theories in $D=4,5$, topological (moduliindependent) U-invariants constructed in terms of (moduli-dependent) central charges and matter charges, and show that, as expected, they coincide with $M_{A D M}^{2}$ or $M_{A D M}^{3 / 2}$ (in the case of the four and five dimensional theories respectively), computed at "fixed scalars".

The situation of black-hole backgrounds preserving more than $1 / 8$ of the original supersymmetry (32 charges) is further explored.

Sections 2 and 3 deal with the four and five dimensional cases respectively, section 4 describes the absolute duality invariants, section 5 describes BPS states preserving more than one supersymmetry and section 6 gives a further classification of BPS states in terms of duality orbits.

\section{Central charges, U-invariants and en- tropy in $D=4$}

In $D=4$, extremal black-holes preserving one supersymmetry correspond to $N$-extended multiplets with

$M_{A D M}=\left|Z_{1}\right|>\left|Z_{2}\right| \cdots>\left|Z_{[N / 2]}\right|$

where $Z_{\alpha}, \alpha=1, \cdots,[N / 2]$, are the proper values of the central charge antisymmetric matrix written in normal form [11]. The central charges $Z_{A B}=-Z_{B A}, A, B=1, \cdots, N$, and matter charges $Z_{I}, I=1, \cdots, n$ are those (modulidependent) symplectic invariant combinations of field strenghts and their duals (integrated over a large two-sphere) which appear in the gravitino and gaugino supersymmetry variations respectively [12], [13], [14]. Note that the total number of vector fields is $n_{v}=N(N-1) / 2+n$ (with the exception of $N=6$ in which case there is an extra singlet graviphoton) 15 .

It was shown in ref. [7] that at the attractor point, where $M_{A D M}$ is extremized, supersymmetry requires that $Z_{\alpha}, \alpha>1$, vanish together with the matter charges $Z_{I}, I=1, \cdots, n$ ( $n$ is the number of matter multiplets, which can exist only for $N=3,4$ )

This result can be used to show that for "fixed scalars", corresponding to the attractor point, the scalar "potential" of the geodesic action [9] 10$]$

$V=-\frac{1}{2} P^{t} \mathcal{M}(\mathcal{N}) P$

is extremized in moduli space. Here $P$ is the symplectic vector $P=\left(p^{\Lambda}, q_{\Lambda}\right)$ of quantized electric and magnetic charges and $\mathcal{M}(\mathcal{N})$ is a symplectic $2 n_{v} \times 2 n_{v}$ matrix whose $n_{v} \times n_{v}$ blocks are given in terms of the $n_{v} \times n_{v}$ vector kinetic matrix $\mathcal{N}_{\Lambda \Sigma}(-\operatorname{Im} \mathcal{N}, \operatorname{Re} \mathcal{N}$ are the normalizations of the kinetic $F^{2}$ and the topological $F^{*} F$ terms respectively) and

$\mathcal{M}(\mathcal{N})=\left(\begin{array}{ll}A & B \\ C & D\end{array}\right)$

with:

$$
\begin{aligned}
& A=\operatorname{Im\mathcal {N}}+\operatorname{ReN} \operatorname{Im} \mathcal{N}^{-1} \operatorname{Re} \mathcal{N} \\
& B=-\operatorname{Re} \mathcal{N} \operatorname{Im} \mathcal{N}^{-1} \\
& C=-\operatorname{Im} \mathcal{N}^{-1} \operatorname{Re} \mathcal{N} \\
& D=\operatorname{ImN}^{-1}
\end{aligned}
$$

The above assertion comes from the important identity, shown in ref. [13], 14 to be valid in all $N \geq 2$ theories:

$$
-\frac{1}{2} P^{t} \mathcal{M}(\mathcal{N}) P=\frac{1}{2} Z_{A B} \bar{Z}^{A B}+Z_{I} \bar{Z}^{I}
$$

Indeed, let us consider the differential relations satisfied by the charges [14]:

$$
\begin{aligned}
\nabla Z_{A B} & =\frac{1}{2} P_{A B C D} \bar{Z}^{C D}+P_{A B I} \bar{Z}^{I} \\
\nabla Z_{I} & =\frac{1}{2} P_{A B I} \bar{Z}^{A B}+P_{I J} \bar{Z}^{J}
\end{aligned}
$$

where the matrices $P_{A B C D}, P_{A B I}, P_{I J}$ are the subblocks of the vielbein of $G / H$ embedded in $U S p(n, n)$ [14:

$\mathcal{P} \equiv L^{-1} \nabla L=\left(\begin{array}{cc}P_{A B C D} & P_{A B J} \\ P_{I C D} & P_{I J}\end{array}\right)$

written in terms of the indices of $H=H_{A u t} \times$ $H_{\text {matter. }}$. By computing the extremum of (2) and using equations (5), (6) we obtain

$P^{A B C D} Z_{A B} Z_{C D}=0 ; \quad Z_{I}=0$

$P_{A B C D}$ being the vielbein of the scalar manifold, completely antisymmetric in its $S U(N)$ indices. 
It is easy to see that in the normal frame these equations imply:

$$
\begin{aligned}
\left.M_{A D M}\right|_{f i x} & \equiv\left|Z_{1}\right| \neq 0 \\
\left|Z_{i}\right| & =0 \quad(i=2, \cdots, N / 2)
\end{aligned}
$$

The main purpose of this section is to provide particular expressions which give the entropy formula as a moduli-independent quantity in the entire moduli space and not just at the critical points. Namely, we are looking for quantities $S\left(Z_{A B}(\phi), \bar{Z}^{A B}(\phi), Z_{I}(\phi), \bar{Z}^{I}(\phi)\right)$ such that $\frac{\partial}{\partial \phi^{i}} S=0, \phi^{i}$ being the moduli coordinates.

These formulae generalize the quartic $E_{7(7)}$ invariant of $N=8$ supergravity [8] to all other cases.

Let us first consider the theories $N=3,4$, where matter can be present [16], [17].

The U-duality groups are, in these cases, $S U(3, n)$ and $S U(1,1) \times S O(6, n)$ respectively (Here we denote by U-duality group the isometry group $G$ acting on the scalars, although only a restriction of it to integers is the proper U-duality group [18]). The central and matter charges $Z_{A B}, Z_{I}$ transform in an obvious way under the isotropy groups

$$
\begin{aligned}
& H=S U(3) \times S U(n) \times U(1) \quad(N=3) \\
& H=S U(4) \times O(n) \times U(1) \quad(N=4)
\end{aligned}
$$

Under the action of the elements of $G / H$ the charges get mixed with their complex conjugate. For $N=3$ :

$$
\begin{aligned}
P^{A B C D} & =P_{I J}=0, P_{A B I} \equiv \epsilon_{A B C} P_{I}^{C} \\
Z_{A B} & \equiv \epsilon_{A B C} Z^{C}
\end{aligned}
$$

Then the variations are:

$$
\begin{aligned}
\delta Z^{A} & =\xi_{I}^{A} \bar{Z}^{I} \\
\delta Z_{I} & =\xi_{I}^{A} \bar{Z}_{A}
\end{aligned}
$$

where $\xi_{I}^{A}$ are infinitesimal parameters of $K=$ $G / H$. Indeed, once the covariant derivatives are known, the variations are obtained by the substitution $\nabla \rightarrow \delta, P \rightarrow \xi$.

With a simple calculation, the U-invariant expression is:

$S=Z^{A} \bar{Z}_{A}-Z_{I} \bar{Z}^{I}$
In other words, $\nabla_{i} S=\partial_{i} S=0$, where the covariant derivative is defined in ref. [14].

Note that at the attractor point $\left(Z_{I}=0\right)$ it coincides with the moduli-dependent potential (2) computed at its extremum.

For $N=4$

$$
\begin{aligned}
P_{A B C D} & =\epsilon_{A B C D} P, \quad P_{I J}=\eta_{I J} \bar{P} \\
P_{A B I} & =\frac{1}{2} \eta_{I J} \epsilon_{A B C D} \bar{P}^{C D J}
\end{aligned}
$$

and the transformations of $K=\frac{S U(1,1)}{U(1)} \times$ $\frac{O(6, n)}{O(6) \times O(n)}$ are:

$$
\begin{aligned}
\delta Z_{A B} & =\frac{1}{2} \xi \epsilon_{A B C D} \bar{Z}^{C D}+\xi_{A B I} \bar{Z}^{I} \\
\delta Z_{I} & =\bar{\xi} \eta_{I J} \bar{Z}^{J}+\frac{1}{2} \xi_{A B I} \bar{Z}^{A B}
\end{aligned}
$$

with $\bar{\xi}^{A B I}=\frac{1}{2} \eta^{I J} \epsilon^{A B C D} \xi_{C D J}$.

There are three $O(6, n)$ invariants given by $I_{1}$, $I_{2}, \bar{I}_{2}$ where:

$$
\begin{aligned}
& I_{1}=\frac{1}{2} Z_{A B} \bar{Z}_{A B}-Z_{I} \bar{Z}^{I} \\
& I_{2}=\frac{1}{4} \epsilon^{A B C D} Z_{A B} Z_{C D}-\bar{Z}_{I} \bar{Z}^{I}
\end{aligned}
$$

and the unique $S U(1,1) \times O(6, n)$ invariant $S$, $\nabla S=0$, is given by:

$S=\sqrt{\left(I_{1}\right)^{2}-\left|I_{2}\right|^{2}}$

At the attractor point $Z_{I}=0$ and $\epsilon^{A B C D} Z_{A B} Z_{C D}=0$, so that $S$ reduces to the square of the BPS mass.

For $N=5,6,8$ the U-duality invariant expression $S$ is the square root of a unique invariant under the corresponding U-duality groups $S U(5,1)$, $O^{*}(12)$ and $E_{7(7)}$. The strategy is to find a quartic expression $S^{2}$ in terms of $Z_{A B}$ such that $\nabla S=0$, i.e. $S$ is moduli-independent.

As before, this quantity is a particular combination of the $H$ quartic invariants.

For $S U(5,1)$ there are only two $U(5)$ quartic invariants. In terms of the matrix $A_{A}^{B}=Z_{A C} \bar{Z}^{C B}$ they are: $(\operatorname{Tr} A)^{2}, \operatorname{Tr}\left(A^{2}\right)$, where

$$
\begin{aligned}
\operatorname{Tr} A & =Z_{A B} \bar{Z}^{B A} \\
\operatorname{Tr}\left(A^{2}\right) & =Z_{A B} \bar{Z}^{B C} Z_{C D} \bar{Z}^{D A}
\end{aligned}
$$


As before, the relative coefficient is fixed by the transformation properties of $Z_{A B}$ under $\frac{S U(5,1)}{U(5)}$ elements of infinitesimal parameter $\xi^{C}$ :

$\delta Z_{A B}=\frac{1}{2} \xi^{C} \epsilon_{C A B P Q} \bar{Z}^{P Q}$

It then follows that the required invariant is:

$S=\frac{1}{2} \sqrt{4 \operatorname{Tr}\left(A^{2}\right)-(\operatorname{Tr} A)^{2}}$

For $N=8$ the $S U(8)$ invariants are:

$$
\begin{aligned}
I_{1} & =(\operatorname{Tr} A)^{2} \\
I_{2} & =\operatorname{Tr}\left(A^{2}\right) \\
I_{3} & =\operatorname{Pf} Z \\
& =\frac{1}{2^{4} 4 !} \epsilon^{A B C D E F G H} Z_{A B} Z_{C D} Z_{E F} Z_{G H}
\end{aligned}
$$

The $\frac{E_{7(7)}}{S U(8)}$ transformations are:

$\delta Z_{A B}=\frac{1}{2} \xi_{A B C D} \bar{Z}^{C D}$

where $\xi_{A B C D}$ satisfies the reality constraint:

$\xi_{A B C D}=\frac{1}{24} \epsilon_{A B C D E F G H} \bar{\xi}^{E F G H}$

One finds the following $E_{7(7)}$ invariant [8]:

$S=\frac{1}{2} \sqrt{4 \operatorname{Tr}\left(A^{2}\right)-(\operatorname{Tr} A)^{2}+32 \operatorname{Re}(\operatorname{Pf} Z)}$

The $N=6$ case is the more complicated because under $U(6)$ the left-handed spinor of $O^{*}(12)$ splits into:

$32_{L} \rightarrow(15,1)+(\overline{15},-1)+(1,-3)+(1,3)$

The transformations of $\frac{O^{*}(12)}{U(6)}$ are:

$$
\begin{aligned}
\delta Z_{A B} & =\frac{1}{4} \epsilon_{A B C D E F} \xi^{C D} \bar{Z}^{E F}+\xi_{A B} \bar{X} \\
\delta X & =\frac{1}{2} \xi_{A B} \bar{Z}^{A B}
\end{aligned}
$$

where we denote by $X$ the $S U(6)$ singlet. The quartic $U(6)$ invariants are:

$$
\begin{aligned}
I_{1} & =(\operatorname{Tr} A)^{2} \\
I_{2} & =\operatorname{Tr}\left(A^{2}\right) \\
I_{3} & =\operatorname{Re}(\operatorname{Pf} Z X) \\
& =\frac{1}{2^{3} 3 !} \operatorname{Re}\left(\epsilon^{A B C D E F} Z_{A B} Z_{C D} Z_{E F} X\right) \\
I_{4} & =(\operatorname{Tr} A) X \bar{X} \\
I_{5} & =X^{2} \bar{X}^{2}
\end{aligned}
$$

The unique $O^{*}(12)$ invariant is:

$$
\begin{aligned}
S & =\frac{1}{2} \sqrt{4 I_{2}-I_{1}+32 I_{3}+4 I_{4}+4 I_{5}} \\
\nabla S & =0
\end{aligned}
$$

Note that at the attractor point $\operatorname{Pf} Z=0, X=0$ and $S$ reduces to the square of the BPS mass.

We note that in the normal frame the transformations of the coset which preserve the normal form of the $Z_{A B}$ matrix belong to $O(1,1)^{3}$ both for $N=6$ and $N=8$ theories. The relevant $O(1,1)^{3}$ transformations can be read out from eqs. (30), (34) going to the normal frame. The ensuing transformations correspond to commuting matrices which are proper, non compact, Cartan elements of the coset algebra of $N=8, N=6$ respectively 20$]$.

\section{The attractor point condition in $D=5$}

In this section we extend the previous analysis to the $D=5$ dimensional case, for theories with $N>2$ supersymmetries. Theories with $N=2$ at $D=5$ have been considered earlier [6] and fixed scalars recently analyzed in great detail [19]. A technical important difference in this case is that although matter charges vanish for fixed scalars, preserving one supersymmetry requires that the eigenvalues of the central charges which are not the BPS mass do not generally vanish at the horizon, but are all equal and fixed in terms of the entropy.

The five dimensional case exhibits analogies and differencies with respect to the four dimensional one. Exactly like in the four dimensional theories, the entropy is given, through the Bekenstein-Hawking relation, by an invariant of the U-duality group over the entire moduli space and its value is given in terms of the modulidependent scalar potential of the geodesic action [9] at the attractor point [5], [6], [7]:

$$
S=\frac{A}{4}=\frac{\pi^{2}}{12} M_{\text {extr }}^{3 / 2}=\frac{\pi^{2}}{12}\left[\frac{\sqrt{3}}{2} V\left(\phi_{f i x}, g\right)\right]^{3 / 4}
$$

where we have used the relation $V_{\text {extr }}=\frac{4}{3} M_{\text {extr }}^{2}$ which is valid for any $N$ in $D=5$, as we will show in the following. Note that, while in the four dimensional case the U-invariant is quartic in the 
charges, in $D=5$ it turns out to be cubic. Furthermore, in five dimensions the automorphism group under which the central charges transform is $U S p(N)$ instead of $S U(N) \times U(1)$ as in the four dimensional theories [14], [15]. As it is apparent from the dilatino susy transformation law, this implies that, at the minimum of the ADM mass, the central charges different from the maximal one do not vanish, contrary to what happens in $D=4$. Indeed in $D=5$ we may perform again the extremization of the geodesic potential as in $D=4$ but, due to the traceless condition of the antisymmetric symplectic representations of the vielbein $P_{A B C D}$ and of the central charges $Z_{A B}$, the analogous equations:

$P^{A B C D} Z_{A B} Z_{C D}=0 ; \quad Z_{I}=0$

do not imply anymore the vanishing of the central charges different from the mass. Here $A, B, \cdots$ are $U S p(N)$ indices and the antisymmetric matrix $Z_{A B}$ satisfies the reality condition:

$\bar{Z}^{A B}=\mathbb{C}^{A C} \mathbb{C}^{B D} Z_{C D}$

$\mathbb{C}^{A B}$ being the symplectic invariant antisymmetric matrix satisfying $\mathbb{C}=-\mathbb{C}^{T}, \mathbb{C}^{2}=-\mathbb{1}$. Let us now consider more explicitly the various five dimensional theories. In $N=4$ matter coupled supergravity [14], the scalar manifold is given by $G / H=\frac{O(5, n)}{O(5) \times O(n)} \times O(1,1)$. The black-hole potential is given by:

$$
\begin{aligned}
V(\phi, q) & =\frac{1}{2} Z_{A B} \bar{Z}^{A B}+2 X^{2}+Z_{I} Z^{I} \\
& =q_{\Lambda}\left(\mathcal{N}^{-1}\right)^{\Lambda \Sigma} q_{\Sigma}
\end{aligned}
$$

where $X$ is the central charge associated to the singlet photon of the $N=4$ theory, $q_{\Lambda} \equiv$ $\int_{S_{3}} \mathcal{N}_{\Lambda \Sigma}{ }^{\star} F^{\Sigma}$ and $\mathcal{N}_{\Lambda \Sigma}$ are the electric charges and vector kinetic matrix respectively. The central charge $Z_{A B}$ can be decomposed in its $\mathbb{C}$ traceless part $\stackrel{\circ}{Z}_{A B}$ and trace part $X$ according to:

$Z_{A B}=\stackrel{\circ}{Z}_{A B}-\mathbb{C}_{A B} X$

This decomposition corresponds to the combination of the five graviphotons and the singlet photon appearing in the gravitino transformation law 14. The matter charges $Z_{I}$ are instead in the vector representation of $O(n)$. Note that in the dilatino transformation law a different combination of the five graviphotons and the singlet photon appears corresponding to the integrated charge:

$$
\begin{aligned}
Z_{A B}^{(\chi)} & \equiv \frac{1}{2}\left(Z_{A B}+3 \mathbb{C}_{A B} X\right) \\
& =\frac{1}{2}\left(\stackrel{\circ}{Z}_{A B}+2 \mathbb{C}_{A B} X\right)
\end{aligned}
$$

The differential relations satisfied by the central and matter charges are:

$$
\begin{aligned}
\nabla Z_{A B} & =P_{I A B} Z^{I}-2 Z_{A B}^{(\chi)} d \sigma \\
& \rightarrow \nabla \stackrel{\circ}{A B}=P_{I A B} Z^{I}-\stackrel{\circ}{Z}_{A B} d \sigma \\
\nabla X & =2 X d \sigma \\
\nabla Z_{I} & =\frac{1}{4}\left(Z_{A B} \bar{P}_{I}^{A B}\right. \\
& \left.+\bar{Z}^{A B} P_{A B I}\right)-Z_{I} d \sigma
\end{aligned}
$$

To minimize the potential, it is convenient to go to the normal frame where $\stackrel{\circ}{Z}_{A B}$ has proper values $e_{1}, e_{2}=-e_{1}$. In this frame, the potential and the differential relations become:

$$
\begin{aligned}
V(\phi, q) & =e_{1}^{2}+e_{2}^{2}+4 X^{2} \\
\nabla e_{1} & =-e_{1} d \sigma+P_{I} Z^{I} \\
\nabla X & =2 X d \sigma
\end{aligned}
$$

where $P_{I} \equiv P_{I 12}$ is the only independent component of the traceless vielbein one-form $P_{I A B}$ in the normal frame. We then get immediately that, in the normal frame, the fixed scalar condition $\frac{\partial V}{\partial \phi}=0$ requires:

$$
\begin{aligned}
Z_{I} & =0 \\
e_{1} & =-e_{2}=-2 X
\end{aligned}
$$

where $e_{i}(i=1,2)$ are the proper values of $\stackrel{\circ}{Z}_{A B}$. It follows that $M_{\text {extr }}=\left|Z_{12 \text { extr }}\right|=\frac{3}{2} e_{1}$ so that

$V_{\text {extr }}=3 e_{1}^{2}=\frac{4}{3} M_{\text {extr }}^{2}$

In the $N=6$ theory, the scalar manifold is $G / H=S U^{\star}(6) / S p(6)$ 14. The central charge 
$Z_{A B}$ can again be decomposed in a $\mathbb{C}$-traceless part $\stackrel{\circ}{Z}_{A B}$ and a trace part $Z$ according to:

$Z_{A B}=\stackrel{\circ}{Z}_{A B}+\frac{1}{3} \mathbb{C}_{A B} X$

The traceless and trace parts satisfy the differential relations:

$$
\begin{aligned}
\nabla \stackrel{\circ}{Z}_{A B} & =\stackrel{\circ}{Z}_{C[A} P_{B] D} \mathbb{C}^{C D} \\
& +\frac{1}{6} \mathbb{C}_{A B} \stackrel{\circ}{Z}_{L M} P^{L M}+\frac{2}{3} X P_{A B} \\
\nabla X & =\frac{1}{4} \stackrel{\circ}{Z}_{A B} P^{A B}
\end{aligned}
$$

where $P_{A B}$ is the $\mathbb{C}$ traceless vielbein of $G / H$. The geodesic potential has the form:

$$
\begin{aligned}
V(\phi, q) & =\frac{1}{2} Z_{A B} Z^{A B}+\frac{4}{3} X^{2} \\
& =q_{\Lambda \Sigma}\left(\mathcal{N}^{-1}\right)^{\Lambda \Sigma, \Gamma \Delta} q_{\Gamma \Delta}
\end{aligned}
$$

where $\Lambda, \Sigma, \cdots=1, \cdots, 6$ are indices in the fundamental representation of $S U^{\star}(6)$, the couple of indices $\Lambda \Sigma$ in the elctric charges $q_{\Lambda \Sigma}$ are antisymmetric and $(\mathcal{N})_{\Lambda \Sigma, \Gamma \Delta}$ is the kinetic matrix of the vector field-strengths $F^{\Lambda \Sigma}$ To perform the minimization of the potential we proceed as in the $N=4$ case going to the normal frame where $\stackrel{\circ}{Z}_{A B}$ has proper values $e_{1}, e_{2}, e_{3}=-e_{1}-e_{2}$. The potential becomes:

$V(\phi, q)=e_{1}^{2}+e_{2}^{2}+\left(e_{1}+e_{2}\right)^{2}+\frac{4}{3} X^{2}$

Moreover, the differential relations (58) take the form:

$$
\begin{aligned}
\nabla e_{1} & =\frac{1}{3}\left(-e_{1}+e_{2}+2 X\right) P_{1} \\
& +\frac{1}{3}\left(e_{1}+2 e_{2}\right) P_{2} \\
\nabla e_{2} & =\frac{1}{3}\left(2 e_{1}+e_{2}\right) P_{1} \\
& +\frac{1}{3}\left(e_{1}-e_{2}+2 X\right) P_{2} \\
\nabla X & =\frac{1}{2}\left(2 e_{1}+e_{2}\right) P_{1}+\frac{1}{2}\left(e_{1}+2 e_{2}\right) P_{2}
\end{aligned}
$$

where $P_{1}, P_{2}, P_{3}=-P_{1}-P_{2}$ are the proper values of the vielbein one-form $P_{A B}$ in the normal frame. Imposing the attractor-point constraint $\frac{\partial V}{\partial \phi}=0$ on the potential we get the following relations among the charges at the extremum:

$$
\begin{aligned}
e_{2} & =e_{3}=-\frac{1}{2} e_{1}=-\frac{4}{3} X \\
V_{\text {extr }} & =\frac{27}{16} e_{1}^{2}=\frac{4}{3} M_{\text {extr }}^{2} .
\end{aligned}
$$

Note that, using eqs. ( 62)-(64), (65)), the mass $e_{1}+\frac{1}{3} X$ satisfies $\frac{\partial}{\partial \phi^{2}}\left(e_{1}+\frac{1}{3} X\right)=0$ at the extremum, with value $M_{\text {extr }}=\frac{9}{8} e_{1}$.

In the $N=8$ supergravity the scalar manifold is $G / H=E_{6(-6)} / S p(8)$ and the central charges sit in the twice antisymmetric, $\mathbb{C}$-traceless, representation of $U S p(8)$ [14. The scalar "potential" of the geodesic action is given by:

$$
\begin{aligned}
V(\phi, g) & =\frac{1}{2} Z_{A B} \bar{Z}^{A B} \\
& =q_{\Lambda \Sigma}\left(\mathcal{N}^{-1}\right)^{\Lambda \Sigma, \Gamma \Delta}(\phi) q_{\Gamma \Delta}
\end{aligned}
$$

where $q_{\Lambda \Sigma} \equiv \int \mathcal{N}_{\Lambda \Sigma, \Gamma \Delta} F^{\Gamma \Delta}$ are the electric charges and $\mathcal{N}_{\Lambda \Sigma, \Gamma \Delta}$ the vector kinetic matrix. The extremum of $V$ can be found by using the differential relation [14]:

$\nabla Z_{A B}=\frac{1}{2} P_{A B C D} \bar{Z}^{C D}$

where $P_{A B C D}$ is the four-fold antisummetric vielbein one-form of $G / H$. One obtains:

$P^{A B C D} Z_{A B} Z_{C D}=0$

To find the values of the charges at the extremum we use the traceless conditions $\mathbb{C}^{A B} P_{A B C D}=0, \mathbb{C}^{A B} Z_{A B}=0$. In the normal frame, the proper values of $Z_{A B}$ are $e_{1}, e_{2}, e_{3}, e_{4}=-e_{1}-e_{2}-e_{3}$ and we take, as independent components of the vielbein, $P_{1}=$ $P_{1234}=P_{5678}, P_{2}=P_{1256}=P_{3478}\left(P_{3456}=\right.$ $\left.P_{1278}=-P_{1}-P_{2}\right)$. In this way, the covariant derivatives of the charges become:

$$
\begin{aligned}
\nabla e_{1} & =\left(e_{1}+2 e_{2}+e_{3}\right) P_{1} \\
& +\left(e_{1}+e_{2}+2 e_{3}\right) P_{2} \\
\nabla e_{2} & =\left(e_{1}-e_{3}\right) P_{1}+\left(-e_{1}-e_{2}-2 e_{3}\right) P_{2} \\
\nabla e_{3} & =\left(-e_{1}-2 e_{2}-e_{3}\right) P_{1}+\left(e_{1}-e_{2}\right) P_{2}
\end{aligned}
$$


Using these relations, the extremum condition of $V$ implies:

$$
\begin{aligned}
e_{2} & =e_{3}=e_{4}=-\frac{1}{3} e_{1} \\
V_{\text {extr }} & =\frac{4}{3} e_{1}^{2}=\frac{4}{3} M_{\text {extr }}^{2}
\end{aligned}
$$

\section{Topological invariants}

In this section we determine the U-invariant expression in terms of which the entropy can be evaluated over the entire moduli space. Our procedure is the same as in ref. [20], namely we compute cubic $\mathrm{H}$-invariants and determine the appropriate linear combination of them which turns out to be U-invariant. The invariant expression of the entropy for $N=4$ and $N=8$ at $D=5$ in terms of the quantized charges was given in [7]. Let us begin with the $N=4$ theory, controlled by the coset $\frac{O(5, n)}{O(5) \times O(n)} \times O(1,1)$. In this case there are three possible cubic $\mathrm{H}$-invariants, namely:

$$
\begin{aligned}
I_{1} & =\frac{1}{2} \stackrel{\circ}{Z}_{A B} \stackrel{\circ}{Z}^{A B} X \\
I_{2} & =Z_{I} Z_{I} X \\
I_{3} & =X^{3}
\end{aligned}
$$

In order to determine the $U \equiv G=O(5, n) \times$ $O(1,1)$-invariant, we set $I=I_{1}+\alpha I_{2}+\beta I_{3}$ and using the differential relations (48) one easily finds that $\partial I=0$ implies $\alpha=1, \beta=0$. Therefore:

$$
I=I_{1}-I_{2}=\left(\frac{1}{2} \stackrel{\circ}{Z}_{A B} \frac{\circ}{Z}^{A B}-Z_{I} Z_{I}\right) X
$$

is the cubic $(O(5, n) \times O(1,1))$-invariant independent of the moduli and the entropy $S=\frac{\pi}{12} M^{\frac{3}{2}}$ is then given as:

$S \sim I^{1 / 2}=\sqrt{\left(\frac{1}{2} Z_{A B} \bar{Z}^{A B}-Z_{I} Z_{I}\right) Z}$

In the $N=6$ theory, where the coset manifold is $S U^{*}(6) / S p(6)$, the possible cubic $S p(6)$ invariants are:

$$
\begin{aligned}
& I_{1}=\operatorname{Tr}(Z \mathbb{C})^{3} \\
& I_{2}=\operatorname{Tr}(Z \mathbb{C})^{2} X \\
& I_{3}=X^{3}
\end{aligned}
$$

Setting as before:

$I=I_{1}+\alpha I_{2}+\beta I_{3}$

the covariant derivative $\partial I$ is computed using the differential relations (58) and the parametrer $\alpha$ and $\beta$ are then determined by imposing $\partial I=0$. Actually, the best way to perform the computation is to go to the normal frame. Using the differential relations (62)- -64 and the expression for the invariants in the normal frame:

$\operatorname{Tr}(Z \mathbb{C})^{3}=-6\left(e_{1}^{2} e_{2}+e_{1} e_{2}^{2}\right)$

$\operatorname{Tr}(Z \mathbb{C})^{2}=4\left(e_{1}^{2}+e_{2}^{2}+e_{1} e_{2}\right)$

the vanishing of $\partial I$ fixes the coefficients $\alpha$ and $\beta$. The final result is:

$I=-\frac{1}{6} \operatorname{Tr}(Z \mathbb{C})^{3}-\frac{1}{6} \operatorname{Tr}(Z \mathbb{C})^{2} X+\frac{8}{27} X^{3}$

and the entropy is:

$S \sim \sqrt{-\frac{1}{6} \operatorname{Tr}(Z \mathbb{C})^{3}-\frac{1}{6} \operatorname{Tr}(Z \mathbb{C})^{2} X+\frac{8}{27} X^{3}}$

Finally, for the $N=8$ theory, described by the coset $E_{6(6)} / S p(8)$, it is well known that there is a unique $E_{6}$ cubic invariant, namely:

$I_{3}(27)=\operatorname{Tr}(Z \mathbb{C})^{3}=Z_{A}^{B} Z_{B}^{C} Z_{C}^{A}$

where the $S p(8)$ indices are raised and lowered by the antisymmetric matrix $\mathbb{C}_{A B}$. Curiously, the $E_{6}$-invariant corresponds to a single cubic $U S p(8)$-invariant. Again, the invariance of $I$ can be best computed in the normal frame where the invariant $(85)$ takes the form:

$$
\begin{aligned}
I_{3}(27) & =\operatorname{Tr}(Z \mathbb{C})^{3}=-2\left(e_{1}^{3}+e_{2}^{3}+e_{3}^{3}+e_{4}^{3}\right) \\
& =6\left(e_{2}^{2} e_{3}+e_{2} e_{3}^{2}+e_{1}^{2} e_{2}+e_{1} e_{2}^{2}\right. \\
& \left.+e_{1}^{2} e_{3}+e_{1} e_{3}^{2}+2 e_{1} e_{2} e_{3}\right)
\end{aligned}
$$

One finds indeed $\partial I=0$ and therefore

$S \sim I^{1 / 2}=\sqrt{\operatorname{Tr}(Z \mathbb{C})^{3}}$

As in $D=4$ the transformations of the coset which preserve the normal form of the $Z_{A B}$ matrix belong to the non compact Cartan subalgebra of $S U^{*}(6)$ and $E_{6(6)}$ for $N=6$ and $N=8$ respectively, which in both cases turns out to be $S O(1,1)^{2}$.

The relevant $O(1,1)^{2}$ transformations can be read out from eqs. (62)-(64), (69) written in the normal frame 21. 


\section{BPS Conditions for Enhanced Super- symmetry}

In this section we will describe U-duality invariant constraints on the multiplets of quantized charges in the case of BPS black holes whose background preserves more than one supersymmetry [22].

We will still restrict our analysis to four and five dimensional cases for which three possible cases exist i.e. solutions preserving $1 / 8,1 / 4$ and $1 / 2$ of the original supersymmetry (32 charges).

The invariants may only be non zero on solutions preserving $1 / 8$ supersymmetry. In dimensions $6 \leq D \leq 9$ black holes may only preserve $1 / 4$ or $1 / 2$ supersymmetry, and no associated invariants exist in these cases.

The description which follows also make contact with the D-brane microscopic calculation, as it will appear obvious from the formulae given below. We will first consider the five dimensional case.

In this case, BPS states preserving $1 / 4$ of supersymmetry correspond to the invariant constraint $I_{3}(27)=0$ where $I_{3}$ was defined in eq. 86. This corresponds to the $E_{6}$ invariant statement that the $\mathbf{2 7}$ is a null vector with respect to the cubic norm. As we will show in a moment, when this condition is fulfilled it may be shown that two of the central charge eigenvalues are equal in modulus. The generic configuration has 26 independent charges.

Black holes corresponding to $1 / 2$ BPS states correspond to null vectors which are critical, namely

$\partial I(27)=0$

In this case the three central charge eigenvalues are equal in modulus and a generic charge vector has 17 independent components.

To prove the above statements, it is useful to compute the cubic invariant in the normal frame, given by:

$$
\begin{aligned}
I_{3}(27) & =\operatorname{Tr}(Z \mathbb{C})^{3} \\
& =6\left(e_{1}+e_{2}\right)\left(e_{1}+e_{3}\right)\left(e_{2}+e_{3}\right) \\
& =6 s_{1} s_{2} s_{3}
\end{aligned}
$$

where:

$$
\begin{aligned}
& e_{1}=\frac{1}{2}\left(s_{1}+s_{2}-s_{3}\right) \\
& e_{2}=\frac{1}{2}\left(s_{1}-s_{2}+s_{3}\right) \\
& e_{3}=\frac{1}{2}\left(-s_{1}+s_{2}+s_{3}\right)
\end{aligned}
$$

are the eigenvalues of the traceless antisymmetric $8 \times 8$ matrix. We then see that if $s_{1}=0$ then $\left|e_{1}\right|=\left|e_{2}\right|$, and if $s_{1}=s_{2}=0$ then $\left|e_{1}\right|=\left|e_{2}\right|=$ $\left|e_{3}\right|$. To count the independent charges we must add to the eigenvalues the angles given by $U S p(8)$ rotations. The subgroup of $U S p(8)$ leaving two eigenvalues invariant is $U S p(2)^{4}$, which is twelve dimensional. The subgroup of $U S p(8)$ leaving invariant one eigenvalue is $U S p(4) \times U S p(4)$, which is twenty dimensional. The angles are therefore $36-12=24$ in the first case, and $36-20=16$ in the second case. This gives rise to configurations with 26 and 17 charges respectively, as promised.

Taking the case of Type II on $T^{5}$ we can choose $s_{1}$ to correspond to a solitonic five-brane charge, $s_{2}$ to a fundamental string winding charge along some direction and $s_{3}$ to Kaluza-Klein momentum along the same direction.

The basis chosen in the above example is S-dual to the D-brane basis usually chosen for describing black holes in Type IIB on $T_{5}$. All other bases are related by U-duality to this particular choice. We also observe that the above analysis relates the cubic invariant to the picture of intersecting branes since a three-charge 1/8 BPS configuration with non vanishing entropy can be thought as obtained by intersecting three single charge $1 / 2$ BPS configurations [31], [32], 33]

By using the $\mathrm{S}$-T-duality decomposition we see that the cubic invariant reduces to $I_{3}(27)=$ $10_{-2} 10_{-2} 1_{4}+16_{1} 16_{1} 10_{-2}$. The 16 correspond to D-brane charges, the 10 correspond to the $5 \mathrm{KK}$ directions and winding of wrapped fundamental strings, the 1 correspond to the N-S five-brane charge.

We see that to have a non-vanishing area we need a configuration with three non-vanishing N$\mathrm{S}$ charges or two D-brane charges and one N-S charge.

Unlike the $4-D$ case, it is impossible to have 
a non-vanishing entropy for a configuration only carrying D-brane charges.

We now turn to the four dimensional case.

In this case the situation is more subtle because the condition for the 56 to be a null vector (with respect to the quartic norm defined through eq. 32 is not sufficient to enhance the supersymmetry. This can be easily seen by going to the normal frame where it can be shown that for a null vector there are not, in general, coinciding eigenvalues. The condition for $1 / 4$ supersymmetry is that the gradient of the quartic invariant vanish.

The invariant condition for $1 / 2$ supersymmetry is that the second derivative projected into the adjoint representation of $E_{7}$ vanish. This means that, in the symmetric quadratic polynomials of second derivatives, only terms in the 1463 of $E_{7}$ are non-zero. Indeed, it can be shown, going to the normal frame for the 56 written as a skew $8 \times 8$ matrix, that the above conditions imply two and four eigenvalues being equal respectively.

The independent charges of $1 / 4$ and $1 / 2$ preserving supersymmetry are 45 and 28 respectively.

To prove the latter assertion, it is sufficient to see that the two charges normal-form matrix is left invariant by $U S p(4) \times U S p(4)$, while the one charge matrix is left invariant by $U S p(8)$ so the $S U(8)$ angles are $63-20=43$ and $63-36=27$ respectively.

The generic $1 / 8$ supersymmetry preserving configuration of the 56 of $E_{7}$ with non vanishing entropy has five independent parameters in the normal frame and $51=63-12 S U(8)$ angles. This is because the compact little group of the normal frame is $S U(2)^{4}$. The five parameters describe the four eigenvalues and an overall phase of an $8 \times 8$ skew diagonal matrix.

If we allow the phase to vanish, the 56 quartic norm just simplifies as in the five dimensional case:

$$
\begin{aligned}
& I_{4}(56)=s_{1} s_{2} s_{3} s_{4} \\
& =\left(e_{1}+e_{2}+e_{3}+e_{4}\right)\left(e_{1}+e_{2}-e_{3}-e_{4}\right) \\
& \times\left(e_{1}-e_{2}-e_{3}+e_{4}\right)\left(e_{1}-e_{2}+e_{3}-e_{4}\right)
\end{aligned}
$$

where $e_{i}(i=1, \cdots, 4)$ are the four eigenvalues. $1 / 4$ BPS states correspond to $s_{3}=s_{4}=0$ while
$1 / 2$ BPS states correspond to $s_{2}=s_{3}=s_{4}=0$.

An example of this would be a set of four Dbranes oriented along 456, 678, 894, 579 (where the order of the three numbers indicates the orientation of the brane). Note that in choosing the basis the sign of the D-3-brane charges is important; here they are chosen such that taken together with positive coefficients they form a BPS object. The first two possibilities $\left(I_{4} \neq 0\right.$ and $\left.I_{4}=0, \frac{\partial I_{4}}{\partial q^{i} \neq 0}\right)$ preserve $1 / 8$ of the supersymmetries, the third $\left(\frac{\partial I_{4}}{\partial q^{2}}=0,\left.\frac{\partial^{2} I_{4}}{\partial q^{2} \partial q^{j}}\right|_{\text {Adj } E_{7}} \neq 0\right) 1 / 4$ and the last $\left(\left.\frac{\partial^{2} I_{4}}{\partial q^{i} \partial q^{j}}\right|_{A d j E_{7}}=0\right) 1 / 2$.

It is interesting that there are two types of $1 / 8$ BPS solutions. In the supergravity description, the difference between them is that the first case has non-zero horizon area. If $I_{4}<0$ the solution is not BPS. This case corresponds, for example, to changing the sign of one of the three-brane charges discussed above. By U-duality transformations we can relate this to configurations of branes at angles such as in 23

Going from four to five dimensions it is natural to decompose the $E_{7} \rightarrow E_{6} \times O(1,1)$ where $E_{6}$ is the duality group in five dimensions and $O(1,1)$ is the extra T-duality that appears when we compactify from five to four dimensions. According to this decomposition, the representation breaks as: $\mathbf{5 6} \rightarrow \mathbf{2 7}_{1}+\mathbf{1}_{-3}+\mathbf{2 7}_{-1}^{\prime}+\mathbf{1}_{3}$ and the quartic invariant becomes:

$$
\begin{aligned}
\mathbf{5 6}^{4} & =\left(\mathbf{2 7 _ { 1 }}\right)^{3} \mathbf{1}_{-3}+\left(\mathbf{2 7 ^ { \prime }}{ }_{-1}\right)^{3} \mathbf{1}_{3}+\mathbf{1}_{3} \mathbf{1}_{3} \mathbf{1}_{-3} \mathbf{1}_{-3} \\
& +\mathbf{2} \mathbf{7}_{1} \mathbf{2} \mathbf{7}_{1} \mathbf{2} \mathbf{7}_{-1}^{\prime} \mathbf{2 7 _ { - 1 } ^ { \prime }}+\mathbf{2} \mathbf{7}_{1} \mathbf{2} \mathbf{7}_{-1}^{\prime} \mathbf{1}_{3} \mathbf{1}_{-3}(93)
\end{aligned}
$$

The $\mathbf{2 7}$ comes from point-like charges in five dimensions and the $\mathbf{2 7 ^ { \prime }}$ comes from string-like charges.

Decomposing the U-duality group into T- and S-duality groups, $E_{7} \rightarrow S L(2, \mathbb{R}) \times O(6,6)$, we find $56 \rightarrow(\mathbf{2}, \mathbf{1 2})+(\mathbf{1}, \mathbf{3 2})$ where the first term corresponds to N-S charges and the second term to D-brane charges. Under this decomposition the quartic invariant (92) becomes $\mathbf{5 6}^{4} \rightarrow \mathbf{3 2}^{4}+$ $\left(\mathbf{1 2 . 1 2}^{\prime}\right)^{2}+\mathbf{3 2}^{2} \mathbf{. 1 2 . 1 2}^{\prime}$. This means that we can have configurations with a non-zero area that carry only D-brane charges, or only N-S charges or both D-brane and N-S charges.

It is remarkable that $E_{7(7)}$-duality gives additional restrictions on the BPS states other than 
the ones merely implied by the supersymmetry algebra. The analysis of double extremal black holes implies that $I_{4}$ be semi-definite positive for BPS states. From this fact it follows that configurations preserving $1 / 4$ of supersymmetry must have eigenvalues equal in pairs, while configuratons with three coinciding eigenvalues are not BPS.

To see this, it is sufficient to write the quartic invariant in the normal frame basis. A generic skew diagonal $8 \times 8$ matrix depends on four complex eigenvalues $z_{i}$. These eight real parameters can be understood using the decomposition [34]:

$$
\begin{aligned}
\mathbf{5 6} & \rightarrow\left(\mathbf{8}_{\mathbf{v}}, \mathbf{2}, \mathbf{1}, \mathbf{1}\right)+\left(\mathbf{8}_{\mathbf{s}}, \mathbf{1}, \mathbf{2}, \mathbf{1}\right)+\left(\mathbf{8}_{\mathrm{c}}, \mathbf{1}, \mathbf{1}, \mathbf{2}\right) \\
& +(\mathbf{1}, \mathbf{2}, \mathbf{2}, \mathbf{2})
\end{aligned}
$$

under

$E_{7(7)} \rightarrow O(4,4) \times S L(2, \mathbb{R})^{3}$

Here $O(4,4)$ is the little group of the normal form and the $(\mathbf{2}, \mathbf{2}, \mathbf{2})$ are the four complex skewdiagonal elements. We can further use $U(1)^{3} \subset$ $S L(2, \mathbb{R})^{3}$ to further remove three relative phases so we get the five parameters $z_{i}=\rho_{i} e^{\mathrm{i} \phi / 4}(i=$ $1, \cdots, 4)$.

The quartic invariant, which is also the unique $S L(2, \mathbb{R})^{3}$ invariant built with the $(\mathbf{2}, \mathbf{2}, \mathbf{2})$, becomes 22:

$$
\begin{array}{ll} 
& I_{4}=\sum_{i}\left|z_{i}\right|^{4}-2 \sum_{i<j}\left|z_{i}\right|^{2}\left|z_{j}\right|^{2} \\
& +4\left(z_{1} z_{2} z_{3} z_{4}+\bar{z}_{1} \bar{z}_{2} \bar{z}_{3} \bar{z}_{4}\right) \\
& =\left(\rho_{1}+\rho_{2}+\rho_{3}+\rho_{4}\right)\left(\rho_{1}+\rho_{2}-\rho_{3}-\rho_{4}\right) \\
\times \quad & \left(\rho_{1}-\rho_{2}+\rho_{3}+\rho_{4}\right)\left(\rho_{1}-\rho_{2}-\rho_{3}+\rho_{4}\right) \\
& +8 \rho_{1} \rho_{2} \rho_{3} \rho_{4}(\cos \phi-1)
\end{array}
$$

The last term is semi-definite negative. The first term, for $\rho_{1}=\rho_{2}=\rho$ becomes:

$$
-\left[4 \rho^{2}-\left(\rho_{3}+\rho_{4}\right)^{2}\right]\left(\rho_{3}-\rho_{4}\right)^{2}
$$

which is negative unless $\rho_{3}=\rho_{4}$. So $1 / 4$ BPS states must have

$\rho_{1}=\rho_{2}>\rho_{3}=\rho_{4}, \quad \cos \phi=1$

For $\rho_{1}=\rho_{2}=\rho_{3}=\rho$, the first term in $I_{4}$ becomes:

$$
-\left(3 \rho+\rho_{4}\right)\left(\rho-\rho_{4}\right)^{3}
$$

so we must also have $\rho_{4}=\rho, \quad \cos \phi=1$ which is the $1 / 2$ BPS condition.

An interesting case, where $I_{4}$ is negative, corresponds to a configuration carrying electric and magnetic charges under the same gauge group, for example a 0-brane plus 6-brane configuration which is dual to a $\mathrm{K}-\mathrm{K}$-monopole plus $\mathrm{K}$ K-momentum [24], [25]. This case corresponds to $z_{i}=\rho e^{\mathrm{i} \phi / 4}$ and the phase is $\tan \phi / 4=e / g$ where $e$ is the electric charge and $g$ is the magnetic charge. Using (92) we find that $I_{4}<0$ unless the solution is purely electric or purely magnetic. In [26] it was suggested that $0+6$ does not form a supersymmetric state. Actually, it was shown in 27 that a $0+6$ configuration can be T-dualized into a non-BPS configuration of four intersecting D-3-branes. Of course, $I_{4}$ is negative for both configurations. Notice that even though these two charges are Dirac dual (and U-dual) they are not S-dual in the sense of filling out an $S L(2, \mathbb{Z})$ multiplet. In fact, the $\mathrm{K}-\mathrm{K}$-monopole forms an $S L(2, \mathbb{Z})$ multiplet with a fundamental string winding charge under S-duality [28]

\section{Duality Orbits fo BPS States Preserving Different Numbers of Supersymmetries}

In this section we give an invariant classification of BPS black holes preserving different numbers of supersymmetries in terms of orbits of the $\mathbf{2 7}$ and the $\mathbf{5 6}$ fundamental representations of the duality groups $E_{6(6)}$ and $E_{7(7)}$ resperctively [29], 30].

In five dimensions the generic orbits preserving $1 / 8$ supersymmetry correspond to the 26 dimensional orbits $E_{6(6)} / F_{4(4)}$ so we may think the generic 27 vector of $E_{6}$ parametrized by a point in this orbit and its cubic norm (which actually equals the square of the black-hole entropy).

The light-like orbit, preserving $1 / 4$ supersymmetry, is the 26 dimensional coset $E_{6(6)} / O(5,4) \odot$ $T_{16}$ where $\odot$ denotes the semidirect product.

The critical orbit, preserving maximal $1 / 2$ supersymmetry (this correspond to $\frac{\partial I_{4}}{\partial q^{2}} \neq 0$ ) correspond to the 17 dimensional space

$\frac{E_{6(6)}}{O(5,5) \odot T_{16}}$ 
In the four dimensional case, we have two inequivalent 55 dimensional orbits corresponding to the cosets $E_{7(7)} / E_{6(2)}$ and $E_{7(7)} / E_{6(6)}$ depending on whether $I_{4}(56)>0$ or $I_{4}(56)<0$. The first case corresponds to $1 / 8$ BPS states whith nonvanishing entropy, while the latter corresponds to non BPS states.

There is an additional 55 dimensional lightlike orbit $\left(I_{4}=0\right)$ preserving $1 / 8$ supersymmetry given by $\frac{E_{7(7)}}{F_{4(4)} \odot T_{26}}$.

The critical light-like orbit, preserving 1/4 supersymmetry, is the 45 dimesnsional coset $E_{7(7)} / O(6,5) \odot\left(T_{32} \oplus T_{1}\right)$

The critical orbit corresponding to maximal $1 / 2$ supersymmetry is described by the 28 dimensional quotient space

$$
\frac{E_{7(7)}}{E_{6(6)} \odot T_{27}}
$$

We actually see that the counting of parameters in terms of invariant orbits reproduces the counting previously made in terms of normal frame parameters and angles. The above analysis makes a close parallel between BPS states preserving different numbers of supersymmetries with timelike, space-like and light-like vectors in Minkowski space.

\section{Acknowledgements}

The results of sections 5 and 6 have been obtained by one of the authors (S. F.) in collaborations with J.M. Maldacena and M. Gunaydin.

\section{REFERENCES}

1. For a review, see for instance: M. J. Duff, R. R. Khuri and J. X. Lu, String solitons, Phys. Rep. 259 (1995) 213; M. J. Duff, KaluzaKlein theory in perspective, in Proceedings of the Nobel Symposium Oskar Klein Centenary, Stockholm, September 1994 (World Scientific, 1995), E. Lindstrom editor, hepth/9410046; G. Horowitz, UCSBTH-96-07, gr-qc/9604051; J. M. Maldacena, Ph.D. thesis, hep-th/9607235; M. Cvetic, UPR-714-T, hep-th/9701152
2. G. Gibbons, in Unified theories of Elementary Particles. Critical Assessment and Prospects, Proceedings of the Heisemberg Symposium, München, West Germany, 1981, ed. by P. Breitenlohner and H. P. Dürr, Lecture Notes in Physics Vol. 160 (Springer-Verlag, Berlin, 1982); G. W. Gibbons and C. M. Hull, Phys. lett. 109B (1982) 190; G. W. Gibbons, in Supersymmetry, Supergravity and Related Topics, Proceedings of the XVth GIFT International Physics, Girona, Spain, 1984, ed. by F. del Aguila, J. de Azcárraga and L. Ibáñez, (World Scientific, 1995), pag. 147; R. Kallosh, A. Linde, T. Ortin, A. Peet and A. Van Proeyen, Phys. Rev. D46 (1992) 5278; R. Kallosh, T. Ortin and A. Peet, Phys. Rev. D47 (1993) 5400; R. Kallosh, Phys. Lett. B282 (1992) 80; R. Kallosh and A. Peet, Phys. Rev. D46 (1992) 5223; A. Sen, Nucl. Phys. B440 (1995) 421; Phys. Lett. B303 (1993) 221; Mod. Phys. Lett. A10 (1995) 2081; J. Schwarz and A. Sen, Phys. Lett. B312 (1993) 105; M. Cvetic and D. Youm, Phys. Rev. D53 (1996) 584; M. Cvetic and A. A. Tseytlin, Phys. Rev. D53 (1996) 5619; M. Cvetic and C. M. Hull, Nucl. Phys. B480 (1996) 296

3. A. Strominger and C. Vafa, Phys. Lett. B379 (1996) 99; C. G. Callan and J. M. Maldacena, Nucl. Phys. B472 (1996) 591; G. Horowitz and A. Strominger, Phys. Rev. Lett. B383 (1996) 2368, hep-th/9602051; R. Dijkgraaf, E. Verlinde, H. Verlinde, Nucl.Phys. B486 (1997) 77; P. M. Kaplan, D. A. Lowe, J. M. Maldacena and A. Strominger. hep-th/9609204; J. M. Maldacena, hepth/9611163

4. L. Susskind, hep-th/9309145; L. Susskind and J. Uglum, Phys. Rev. D50 (1994) 2700; F. Larsen and F. Wilczek, Phys. Lett. B375 (1996) 37

5. S. Ferrara, R. Kallosh and A. Strominger, Phys. Rev. D52 (1995) 5412; A. Strominger, Phys. Lett. B383 (1996) 39

6. S. Ferrara and R. Kallosh, Phys. Rev. D54 (1996) 1514

7. S. Ferrara and R. Kallosh, Phys. Rev. D54 (1996) 1525 
8. R. Kallosh and B. Kol, Phys. Rev. D53 (1996) 5344

9. P. Breitenlohner, D. Maison and G. W. Gibbons, Commun. Math. Phys. 120 (1988) 295; G. W. Gibbons, R. Kallosh and B. Kol, Phys. Rev. Lett. 77 (1996) 4992

10. S. Ferrara, G. W. Gibbons and R. Kallosh, hep-th/9702103

11. S. Ferrara, C. Savoy and B. Zumino, Phys. Lett. 100B (1981) 393

12. A. Ceresole, R. D'Auria and S. Ferrara, in " $S$ Duality and Mirror symmetry", Nucl. Phys. (Proc. Suppl.) B46 (1996) 67, ed. E. Gava, K. S. Narain and C. Vafa

13. L. Andrianopoli, R. D'Auria and S. Ferrara, hep-th/9608015, to appear in International Journal of Modern Physics A

14. L. Andrianopoli, R. D'Auria and S. Ferrara, hep-th/9612105

15. E. Cremmer in "Supergravity '81", ed. by S. Ferrara and J. G. Taylor, Pag. 313; B. Julia in "Superspace \&S Supergravity", ed. by S. Hawking and M. Rocek, Cambridge (1981) pag. 331; M. Günaydin, G. Sierra and P.K. Townsend, Phys. Lett. B 133 (1983) 72; M. Günaydin and C. Sacliog̃lu, Comm. Math. Phys. 87 (1982) 159

16. L. Castellani, A. Ceresole, R. D'Auria, S. Ferrara, P. Fré and E. Maina, Nucl. Phys. B286 (1986) 317

17. E. Bergshoeff, I. G. Koh and E. Sezgin, Phys. Lett. 155B (1985) 71; M. de Roo and F. Wagemans, Nucl. Phys. B262 (1985) 644

18. C. M. Hull and P. K. Townsend, Nucl. Phys. B451 (1995) 525

19. A. Chou, R. Kallosh, J. Rahmfeld, S.J. Rey, M. Shmakova and W.K. Wong, hepth/9704142

20. L. Andrianopoli, R. D'Auria and S. Ferrara, Phys. Lett. B 403 (1997) 12, hep-th/9703156

21. L. Andrianopoli, R. D'Auria and S. Ferrara, hep-th/9705024

22. S. Ferrara and J.M. Maldacena, hepth/9706097

23. V. Balasubramanian, F. Larsen and R. Leigh, hep-th/9704143

24. R. Khuri and T. Ortin, Phys. Lett. B $\mathbf{3 7 3}$ (1996) 56, hep-th/9512178
25. H. Sheinblatt, hep-th/9705054

26. J. Polchinski, hep-th/9611050

27. W. Taylor, hep-th/9705116

28. A. Sen, hep-th/9705212

29. S. Ferrara and M. Günaydin, hep-th/9708025

30. H. Lü, C.N. Pope and K.S. Stelle, hepth/9708109

31. M. Berkooz, M.R. Douglas and R.G. Leigh, Nucl. Phys. B 480 (1996) 265

32. V. Balasubramanian, F. Larsen and R.G. Leigh, hep-th/9704143

33. H. Lü, C.N. Pope, T.A. Tran and K.W. Xu, hep-th/9708055

34. L. Andrianopoli, R. D'Auria, S. Ferrara, P. Fré, M. Trigiante, hep-th/9707087 